\title{
Effects of mirror feedback during balanced exercise performance in the old people with mild cognitive impairment
}

\author{
Haemi Jee* \\ Department of Physical Therapy, Namseoul University, Cheonan, Korea
}

Unbalanced movements between the bilateral sides may lead to dyskinesia and reduced motor function. The upper limbs are the most complex joints with different rate of usage between the limbs. The dominant side is repeatedly used more than the contralateral side for better dexterity and musculoskeletal utility. Such imbalance between the two bilateral sides may lead to further difference in movement ability. This study aimed to observe the feasibility of the mirror feedback method in the upper limb movements in the old people with mild cognitive impairment. Twenty-seven-old people with history of stroke were selected. Motion sensors were used to assess the location and motion of two limbs in synchronized lateral raise that include the abduction and adduction motions during the mirror feedback and no feedback trials. The results of the mirror feedback showed comparatively similar motions between the left and right upper limbs. The results of the study may indicate possible recommendation of mirror feedback method for synchronizing exercise motion of the upper limbs for the old people with mild cognitive impairment.

Keywords: Cognitive impairment, Old people, Mirror feedback

\section{INTRODUCTION}

Asymmetry in movements and strength are common since most of the people have both the dominant and nondominant sides with different rate of utilization of either side (Yoshizaki et al., 2009). Evaluation of the asymmetric movements between two equivalent sides may provide vital information of the physiological state of a person. For example, comparisons between the strength of the quadriceps and hamstrings have utilized to predict possible fall in the frail or old people populations. Asymmetric balance also has been related to deter of the functional performance in the athletes (Hosseinimehr et al., 2015; Lang et al., 2017; Maly et al., 2015; Sachlikidis and Salter, 2007; Yoshizaki et al., 2009). In addition, the comparative asymmetric results between the left and right sides of the body also have been used to observe or prevent progression into worsening of pathological disorder of the musculo- skeletal system (do Rosário, 2014; Hosseinimehr et al., 2015; Lang et al., 2017; Maly et al., 2015; Matsuki et al., 2011; Yoshizaki et al., 2009).

Functional deterioration with dyskinesia has been previously reported especially in the old people with history of cognitive event such as stroke. It may be important to obtain proper balance between the movements of the left and right sides of the body with equilateral strength and motion to maintain functionality in the old people. Progressively decreasing activity level with ongoing deterioration of various bodily systems including neurological and musculoskeletal systems may lead to greater asymmetry in balance between the equilateral sides of the body in the old people with previous neurological event especially stroke. Various reports on the old people have been shown to maintain or even improve deteriorations of the bodily systems through properly and regularly performed physical activity that includes both aerobic and re-
${ }^{*}$ Corresponding author: Haemi Jee (iD https://orcid.org/0000-0002-9066-1472 Department of Physical Therapy, Namseoul University, 91 Daehak-ro, Seonghwan-eup, Seobuk-gu, Cheonan 31020, Korea E-mail:wlgpal9@gmail.com

Received: December 28, 2019 / Accepted: January 27, 2020
This is an Open Access article distributed under the terms of the Creative Commons Attribution Non-Commercial License (https://creativecommons.org/licenses/by-nc/4.0/) which permits unrestricted non-commercial use, distribution, and reproduction in any medium, provided the original work is properly cited. 
sistant exercise regime.

Participating in proper regime of physical activity regularly has been known to be vital. Clinics may instruct on the proper performance posture to the patients. However, the patient follow-up rates to the clinics that provide customized exercise program based on assessment results have been reported to be slow (Park et al., 2014). Although supervised exercise is more effective, it may be more practical to provide effective home exercise training methods that could be easily utilized by the old people with history of brain lesion (Park et al., 2014; Tsekoura et al., 2018).

Utilizing a mirror for providing information for proper movement during various physical activities has long been recommended. Despite of its popularity in various fitness settings, there have been mixed reports on the advantageous nature of the mirror on the motor performance (Halperin et al., 2016). For example, a study which observed balance exercise in the old people did not show considerable improvement in balance (Vaillant et al., 2004). Due to the mixed results on the mirror feedback, it may be necessary to observe the feasibility in the old people with mild cognitive impairment (Crum et al., 1993).

Therefore, the aim of the study was to compare and observe commonly practiced strength exercise that the aged people with previous cognitive event of stroke could use regularly in a manner that provides feedback for balanced exercise motions. Mirror exercise has been utilized to provide feedback to the exerciser oneself for improving neurological function with balanced usage between the left and right sides of the body. In this study, one of the most commonly practiced exercise regime, lateral raises, were conducted by body upper limbs in a synchronized fashion with and without the mirror feedback during exercises (Hurd et al., 2013; Lang et al., 2017). Performance was assessed by comparing synchronized motion between the left and right limbs by angle $\left(^{\circ}\right)$ for the positional comparison and angular acceleration $\left({ }^{\circ} / \mathrm{sec}^{2}\right)$ for the motion comparison.

\section{MATERIALS AND METHODS}

\section{Subjects}

The study purpose and procedure were performed according to the Declaration of Helsinki. The subjects were recruited through person-to-person referrals. Prior to the tests, all participants were fully informed of the purpose and procedure of the experiment. The subjects gave verbal and written informed content to participate in the study. In addition, the subjects with excessive or clinically diagnosed shoulder pain were excluded. The Korean
Mini-Mental State Examination (K-MMSE) test scores, the subjects with K-MMSE scores between 19 and 23 (Crum et al., 1993) were selected. Twenty-seven, seven males and twenty females, aged between 65 to 82 years with mean age of $68 \pm 6.34$ years and mean body mass index of $25 \pm 4.37 \mathrm{~kg} / \mathrm{m}^{2}$ participated in performing the lateral raise with two dumbbells held on both hands. The subjects regularly participated in low-intensity physical activity $3.1 \pm$ 1.12 days per week and $34.3 \pm 19.31$ minutes per day on the average. The pain scale was $7.0 \% \pm 9.11 \%$ and the disability scale was $2.3 \% \pm 4.4 \%$. The average dominant handedness score was 9.2 out of scales from -10 to 10 . The line bisection test showed right-sided bias results as with previous stroke patients (Jee, 2019). All participants were right-handed. As for the negative and positive values in the dominant handedness score, the positive values indicated right handedness and negative score indicated left-handedness.

\section{Experimental procedure}

The lateral raises were utilized in this study as the exercise method according to previous study (Noble et al., 1983). The rate of perceived exercise or rating of perceived exertion (RPE) was asked from the subjects immediately after performing lateral raise for the subjective intensity of the exercise with Borg scale ranging from zero to 10 (Andersen et al., 2010; Noble et al., 1983). The subjects were given instructions to restrain from excessive physical activity and consumption of meal since a day prior to the assessment.

\section{Questionnaires}

Just prior to the assessment, the subjects were asked of information that included date of birth, bodily weight $(\mathrm{kg})$, and height $(\mathrm{cm})$. In addition to the general inquiry on the physical status, medical condition, current health status, and performance of regular exercise were asked. Additional inquiry on the dominant hand, shoulder pain, line bisection test, and disability status were also made. Mental status by K-MMSE was also given to assess the cognitive health status of the subjects as previously conducted to assess mild cognitive impairment (Crum et al., 1993; Hara et al., 1984; Jee and Park, 2019). The Shoulder Pain Disability Index (SPADI) was used to assess the shoulder pain and disability (Noble et al., 1983). The subjects with excessive shoulder pain were excluded based on the interview and SPADI scores ( $>13$ points) (Roach et al., 1991). The Edinburgh Inventory tool was used to assess dominant handedness (Oldfield, 1971). All subjects showed right handedness (Veale, 2014). 


\section{Exercise performance}

Prior calculation of one-repetition maximum (1RM) was made based on the reports of prior studies (Dankel et al., 2019; Jee, 2019). The trial-and-error method was also applied to reflect the actual dumwbbell weight appropriate for each subject (Dankel et al., 2019; Jee, 2019). Low intensity ( $40 \%$ or less of 1RM) was applied as the intensity of the resistant exercise and the lowest dumbbell weight of $1 \mathrm{~kg}$ per arm was given to all subjects.

Just prior to the lateral raise test, the subjects were given the dumbbells for familiarity of the dumbbells. The assessor explained and showed each subject of the proper abduction and adduction motions of the lateral raises as previous studies (Andersen et al., 2010; Jee, 2019). Each subject was asked to perform the same lateral raise motions to prevent injury or abnormal motions during actual assessment. The subjects were asked to slowly raise the dumbbells on each hand at same slow and synchronize manner without a jerking motion. The lateral raises composed of abduction and adduction motions were asked to perform at least 10 times. The subjects were informed to terminate the lateral raises voluntarily anytime they feel that they are unable to continue with the motions. The Borg scale (RPE: 1 to 10) was also used to assess the perceived intensity (Jee, 2019).

Each subject was asked to stand up-right behind the predrawn line on the floor. Mirror was not utilized for the first nonmirror feedback trial. As for the second mirror feedback trial, procedure was conducted as reported by previous study (Halperin et al., 2016). A full-body sized mirror was placed $1 \mathrm{~m}$ away from the subject for a full-body observation by the subjects.

\section{Motion assessment}

Previously evaluated set of devices for reliability composed of two synchronized sensors was used to the assessment (Yun and Bachmann, 2006). Two synchronized devices that are connected to a computer were attached to distal ends of both the left and right radioulnar joints. The devices simultaneously retrieved and sent the movement information to the computer via Bluetooth by obtaining the initial angle $\left(^{\circ}\right)$ and acceleration $\left(\% / \mathrm{sec}^{2}\right)$ every tenth of a second (Jee, 2019). As for the purpose of the study, the minimum (starting position of lateral raise), maximum (ending position of lateral raise), and $45^{\circ}$ angles $\left(^{\circ}\right)$ and accelerations $\left({ }^{\circ} / \mathrm{sec}^{2}\right)$ were selected for the comparative analysis (Jee, 2019).

The first trial of the lateral raises was conducted without observing the reflection of the subject self. Seven days were given to each subject prior to conducting the second bout of exercise with mirror for mirror feedback of the exercise performed. Identical proce- dural protocols were applied for the second assessment trial (Jee and Park, 2019).

\section{Statistical analysis}

The sample size of the study was initially determined using previous studies for performing lateral raises. The normality assessment was first performed via Shapiro-Wilk test prior to the performing comparative analysis. The initial assessment test indicated normal distribution of the obtained data. In order to compare two sets of data, One-way analysis of variance was performed. The results were given in means with standard deviations. Statistical significance was accepted for the $P$-value of 0.05 .

\section{RESULTS}

The assessment results were compared between left and right upper limbs. One set of results was with the mirror feedback and the other set of results was without the mirror feedback.

The nonmirror feedback comparisons showed significant differences toward the end of the adduction motion of $45^{\circ}\left(48.96^{\circ} \pm\right.$ $8.48^{\circ}$ vs. $\left.43.40^{\circ} \pm 7.18^{\circ}, P=0.03\right)$ and end of the adduction motion $\left(6.76^{\circ} \pm 5.46^{\circ}\right.$ vs. $\left.10.80^{\circ} \pm 4.62^{\circ}, P=0.02\right)$. In addition, although significance was not shown $(P=0.07)$, the greatest angular difference was shown at the end of the abduction motion of $90^{\circ}$. As for the acceleration comparisons $(\% / \mathrm{sec})$, significant difference was shown between left and right throughout the lateral raise motion. At the start of the abduction motion, the right side accelerated significantly faster than the left side $\left(0.68 \pm 0.47 \% / \mathrm{sec}^{2}\right.$ vs. $1.26 \pm$ $\left.0.47^{\circ} / \mathrm{sec}^{2}, P<0.01\right)$. The end of the abduction motion sides $(2.98 \pm$ $1.35 \% \mathrm{sec}^{2}$ vs. $\left.2.17 \pm 1.06^{\circ} / \mathrm{sec}^{2}, P=0.04\right)$ and adduction motion $\left(0.76 \pm 0.49^{\circ} / \mathrm{sec}^{2}\right.$ vs. $\left.1.18 \pm 0.34 \% / \mathrm{sec}^{2}, P<0.01\right)$ also showed significant differences between the two sides.

The mirror feedback comparisons of the position by angles showed significant difference between the limbs at the start of abduction motion $\left(9.05^{\circ} \pm 2.94^{\circ}\right.$ vs. $12.45^{\circ} \pm 5.41^{\circ}, P=0.02$ ) (Table 1$)$. As for the acceleration of the limbs, the two sides showed significant difference at the end of the adduction motion $\left(1.17 \pm 0.39^{\circ} / \mathrm{sec}^{2}\right.$ vs. $0.77 \pm 0.46 \% \mathrm{sec}^{2}, P=0.01$ ) (Table 2 ).

\section{DISCUSSION}

Comparative differences in strength and motion of coronary or sagittal halves of the body have long been utilized to predict further functional disability or development of musculoskeletal disorder. Such asymmetric musculoskeletal problems could worsen 
Table 1. Comparisons of the location by angle $\left(^{\circ}\right)$ of the left and right limbs during abduction and adduction motions

\begin{tabular}{|c|c|c|c|c|c|}
\hline Fixed angles $\left({ }^{\circ}\right)$ & Left $\operatorname{limb}\left({ }^{\circ}\right)$ & Right $\operatorname{limb}\left({ }^{\circ}\right)$ & Mean differences & $F$ & $P$-value \\
\hline \multicolumn{6}{|c|}{ Mirror feedback comparisons } \\
\hline Start abduction & $9.05 \pm 2.94$ & $12.45 \pm 5.41$ & -3.40 & 10.9 & $0.02^{*}$ \\
\hline $45^{\circ}$ abduction & $34.95 \pm 0.56$ & $34.95 \pm 9.01$ & -3.98 & 45.5 & 0.06 \\
\hline End abduction & $94.21 \pm 11.04$ & $92.35 \pm 9.34$ & 1.85 & 0.5 & 0.57 \\
\hline $45^{\circ}$ adduction & $44.69 \pm 0.61$ & $47.66 \pm 11.61$ & -2.96 & 18.2 & 0.26 \\
\hline End adduction & $7.60 \pm 11.61$ & $9.96 \pm 4.77$ & -2.36 & 1.9 & 0.17 \\
\hline \multicolumn{6}{|c|}{ Nonmirror feedback comparisons } \\
\hline Start abduction & $9.92 \pm 4.69$ & $11.59 \pm 4.53$ & -1.67 & 0.12 & 0.26 \\
\hline $45^{\circ}$ abduction & $38.47 \pm 6.99$ & $35.42 \pm 6.01$ & 3.05 & 1.4 & 0.15 \\
\hline End abduction & $90.38 \pm 7.11$ & $96.18 \pm 11.95$ & -5.81 & 8.4 & 0.07 \\
\hline $45^{\circ}$ adduction & $48.96 \pm 8.48$ & $43.40 \pm 7.18$ & 5.56 & 0.4 & $0.03^{*}$ \\
\hline End adduction & $6.76 \pm 5.46$ & $10.80 \pm 4.62$ & -4.04 & 0.6 & $0.02^{*}$ \\
\hline
\end{tabular}

Values are presented as mean \pm standard deviation.

${ }^{*} P<0.05$.

Table 2. Comparisons of the motion by acceleration during abduction and adduction motions

\begin{tabular}{|c|c|c|c|c|c|}
\hline Fixed angles $\left({ }^{\circ}\right)$ & Left $\operatorname{limb}\left(\% / \sec ^{2}\right)$ & Right $\operatorname{limb}\left(\% / \sec ^{2}\right)$ & Mean differences & $F$ & $P$-value \\
\hline \multicolumn{6}{|c|}{ Mirror feedback comparisons } \\
\hline Start abduction & $1.09 \pm 0.41$ & $0.85 \pm 0.65$ & 0.24 & 5.0 & 0.17 \\
\hline $45^{\circ}$ abduction & $1.23 \pm 0.83$ & $1.40 \pm 0.77$ & -0.14 & 0.4 & 0.58 \\
\hline End abduction & $2.72 \pm 1.43$ & $2.46 \pm 1.12$ & 0.25 & 1.3 & 0.54 \\
\hline $45^{\circ}$ adduction & $1.50 \pm 1.00$ & $1.04 \pm 1.04$ & 0.47 & 0.5 & 0.16 \\
\hline End adduction & $1.17 \pm 0.39$ & $0.77 \pm 0.46$ & 0.40 & 0.8 & $0.01 *$ \\
\hline \multicolumn{6}{|c|}{ Nonmirror feedback comparisons } \\
\hline Start abduction & $0.68 \pm 0.47$ & $1.26 \pm 0.47$ & -0.58 & 0.2 & $<0.01^{*}$ \\
\hline $45^{\circ}$ abduction & $1.18 \pm 0.71$ & $1.44 \pm 0.87$ & -0.26 & 10.0 & 0.30 \\
\hline End abduction & $2.98 \pm 1.35$ & $2.17 \pm 1.06$ & 0.81 & 3.1 & $0.04^{*}$ \\
\hline $45^{\circ}$ adduction & $1.37 \pm 1.28$ & $1.17 \pm 0.73$ & 0.20 & 3.0 & 0.56 \\
\hline End adduction & $0.76 \pm 0.49$ & $1.18 \pm 0.34$ & -0.43 & 3.2 & $<0.01^{*}$ \\
\hline
\end{tabular}

Values are presented as mean \pm standard deviation.

${ }^{*} P<0.05$.

through various internal and external factors such as personal movement habit, incorrectly performed exercise, posture, neurological dysfunction, or aging. Imbalance between the sides may further aggravate the existing problem to accelerate the asymmetry between the sides. In order to prevent and properly manage the development of such imbalance, correct assessment with optimal exercise protocol could be recommended.

In order to provide an optimal method for home exercise, mirror feedback method was compared to regular exercise regime without the mirror feedback in the old people with history of stroke. A stroke event may led to a permanent lesion in the hemisphere. It would affect neurological and musculoskeletal system and further worsen the dysfunction related to the systems (Choi et al., 2015;
Nijboer et al., 2013). Lateral raises were performed by the subjects and the motions were recorded by position and acceleration. The comparative results showed that the significant differences in position and motion were shown while the subjects were not provided with the mirror feedback of their motion.

Previous study on the comparison between the left and right upper limb motion by lateral raises show that the starting position and the final position of the abduction motion showed greatest different difference in motion between the limbs (Jee, 2019; Jee and Park, 2019). The results of this study also showed significantly different position and motion during such locations. Such dynamitic motion during the initial and final phases of the motion was also reported in previous studies (Jee, 2019; Yoshizaki et al., 2009). 
The range of motion was greater for the nondominant arm or the left arm than the dominant right arm for both results in this study. Previous study also showed greater minimum and maximum ranges of motion despite the instruction to start at $10^{\circ}$ from the body and end at $90^{\circ}$ or parallel to the shoulder line (Jee, 2019; Jee and Park 2019). Such results may be due to the better dexterity with greater control of the dominant side than the nondominant side (Bagesteiro et al., 2002). Such comparative differences were also shown with the acceleration of the limbs (Jee, 2019; Sachlikidis and Salter, 2007).

Greater changes in motion were shown during the end of the abduction motion or at the $90^{\circ}$ point when the raising motion of the limbs was terminating. In addition, the speed either accelerated or decelerated more in the dominant arm than the nondominant arm in both assessment results. Such results were also shown in previous studies. Previous studies explained phenomenon with the speed and accuracy trade off. Superior ability to control the motion by the dominant side allowed small sacrifice of accuracy to obtain speed. Previous studies explained of better dexterity of the dominant side by the usage rate of the dominant side with greater torque efficient movements for greater accuracy and control (Lang et al., 2017). Greater dexterity of the dominant side may be supported by various factors such as greater strength, power, and coordination ability of various segments of the body in addition to motor control ability (Dayanidhi et al., 2005; McGrath et al., 2016; Sachlikidis and Salter, 2007; Wickham et al., 2010).

As for the mirror feedback to nonmirror feedback comparisons, the results of this study showed that the mirror feedback method provided comparatively stable motions during the abduction and adduction phases of a full lateral raise. The overall mean differences of the angle by position and motion by acceleration of the mirror feedback method were comparatively less than the non-selffeedback method. Significant differences were shown more for the comparative results of the nonmirror feedback method. Such results of the study indicate using a mirror during the lateral raise exercise lead to less asymmetrical movements by location and motion. Such improvement in movements using the mirror feedback method has been reported in previous studies with old aged groups with various cognitive complications (Kim et al., 2016; Louw et al., 2017).

Observational studies reported of excitability of the primary motor cortex during mirror therapy via functional magnetic resonance images indicating positive stimulation in those with cognitive disability (Kim et al., 2016; Luft et al., 2002). Age-related deterioration lead to reduced motor coordination ability in older adults. Muscle atrophy with neurological deterioration has been reported to accelerate with the progression of aging. Therefore, such deterioration accelerates with a stroke event. Participation of regularly performed physical activity including resistance training has been reported to maintain or deter progression of such musculoskeletal deterioration with activation of the neurological motor function. However, proper exercise is recommended to prevent imbalance between two sides of the body especially in the early phase of the musculoskeletal abnormality to prevent progression into clinical state of dysfunction (Lang et al., 2017; Maly et al., 2015; Yoshizaki et al., 2009). The self-feedback method provided better control over the limbs to reduce positional and movement differences between the two sides. Therefore, based on the results of this study, old people with mild cognitive deterioration or lesion such as by stroke should utilize self-feedback method by utilizing a mirror to reflect their motion during resistant training.

\section{CONFLICT OF INTEREST}

No potential conflict of interest relevant to this article was reported.

\section{ACKNOWLEDGMENTS}

This work was supported by the Ministry of Education of the Republic of Korea and the National Research Foundation of Korea (NRF-2016S1A5A8017498).

\section{REFERENCES}

Andersen LL, Andersen CH, Mortensen OS, Poulsen OM, Bjørnlund IB, Zebis MK. Muscle activation and perceived loading during rehabilitation exercises: comparison of dumbbells and elastic resistance. Phys Ther 2010;90:538-549.

Bagesteiro LB, Sainburg RL. Handedness: dominant arm advantages in control of limb dynamics. J Neurophysiol 2002;88:2408-2421.

Choi M, Yoo J, Shin S. Lee W. The effects of stepper exercise with visual feedback on strength, walking, and stair climbing in individuals following stroke. J Phys Ther Sci. 2015;27:1861-1864.

Crum RM, Anthony JC, Bassett SS, Folstein MF. Population-based norms for the Mini-Mental State Examination by age and educational level. JAMA 1993;269:2386-2391.

Dankel SJ, Bell ZW, Spitz RW, Wong V, Viana RB, Chatakondi RN, Buckner SL, Jessee MB, Mattocks KT, Mouser JG, Abe T, Loenneke JP. Assessing 
differential responders and mean changes in muscle size, strength, and the cross-over effect to two distinct resistance training protocols. Appl Physiol Nutr Metab 2019 Sep 25 [Epub]. https://doi.org/10.1139/apnm2019-0470.

Dayanidhi S, Orlin M, Kozin S, Duff S, Karduna A. Scapular kinematics during humeral elevation in adults and children. Clin Biomech (Bristol, Avon) 2005;20:600-606.

do Rosário JL. Photographic analysis of human posture: a literature review. J Bodyw Mov Ther 2014;18:56-61.

Halperin I, Hughes S, Panchuk D, Abbiss C, Chapman DW. The effects of either a mirror, internal or external focus instructions on single and multi-joint tasks. PLoS One 2016;11:e0166799.

Hara H, Moriki T, Matsusaki K, Hiroi M, Hashimoto M, Yamane T. Distribution pattern of DNA template activity in the developing cerebellar cortex in rats and its alteration after transplacental administration of ethylnitrosourea. Acta Pathol Jpn 1984;34:489-497.

Hosseinimehr SH, Anbarian M, Norasteh AA, Fardmal J, Khosravi MT. The comparison of scapular upward rotation and scapulohumeral rhythm between dominant and non-dominant shoulder in male overhead athletes and non-athletes. Man Ther 2015;20:758-762.

Hurd WJ, Morrow MM, Kaufman KR. Tri-axial accelerometer analysis techniques for evaluating functional use of the extremities. J Electromyogr Kinesiol 2013;23:924-929.

Jee H. Feasibility of a set of wrist-worn novice devices for dual motion comparison of the upper limbs during lateral raise motions. J Exerc Rehabil 2019;15:531-536.

Jee H, Park J. Gender comparisons of asymmetric lateralization in neurodegenerative disorder patients via E-Pen based cognitive assessment system. Iran J Public Health 2019;48:289-298.

Kim MK, Ji SG, Cha HG. The effect of mirror therapy on balance ability of subacute stroke patients. Hong Kong Physiother J 2016;34:27-32.

Lang CE, Waddell KJ, Klaesner JW, Bland MD. A method for quantifying upper limb performance in daily life using accelerometers. J Vis Exp. 2017;(122). https://doi.org/10.3791/55673.

Louw A, Puentedura EJ, Reese D, Parker P, Miller T, Mintken PE. Immediate effects of mirror therapy in patients with shoulder pain and decreased range of motion. Arch Phys Med Rehabil 2017;98:1941-1947.

Luft AR, Smith GV, Forrester L, Whitall J, Macko RF, Hauser TK, Goldberg AP, Hanley DF. Comparing brain activation associated with isolated upper and lower limb movement across corresponding joints. Hum Brain Mapp 2002;17:131-140.

Maly T, Zahalka F, Mala L, Cech P. The bilateral strength and power asym- metries in untrained boys. Open Med (Wars) 2015;10:224-232.

Matsuki K, Matsuki KO, Mu S, Yamaguchi S, Ochiai N, Sasho T, Sugaya $\mathrm{H}$, Toyone T, Wada Y, Takahashi K, Banks SA. In vivo 3-dimensional analysis of scapular kinematics: comparison of dominant and nondominant shoulders. J Shoulder Elbow Surg 2011;20:659-665.

McGrath TM, Waddington G, Scarvell JM, Ball NB, Creer R, Woods K, Smith $\mathrm{D}$. The effect of limb dominance on lower limb functional performance--a systematic review. J Sports Sci 2016;34:289-302.

Noble BJ, Borg GA, Jacobs I, Ceci R, Kaiser P. A category-ratio perceived exertion scale: relationship to blood and muscle lactates and heart rate. Med Sci Sports Exerc 1983;15:523-528.

Oldfield RC. The assessment and analysis of handedness: the Edinburgh inventory. Neuropsychologia 1971;9:97-113.

Park CH, Elavsky S, Koo KM. Factors influencing physical activity in older adults. J Exerc Rehabil 2014;10:45-52.

Roach KE, Budiman-Mak E, Songsiridej N, Lertratanakul Y. Development of a shoulder pain and disability index. Arthritis Care Res 1991;4:143149.

Sachlikidis A, Salter C. A biomechanical comparison of dominant and non-dominant arm throws for speed and accuracy. Sports Biomech 2007;6:334-344.

Tsekoura M, Billis E, Tsepis E, Dimitriadis Z, Matzaroglou C, Tyllianakis M, Panagiotopoulos E, Gliatis J. The effects of group and home-based exercise programs in elderly with sarcopenia: a randomized controlled trial. J Clin Med 2018;7:480.

Vaillant J, Vuillerme N, Janvy A, Louis F, Juvin R, Nougier V. Mirror versus stationary cross feedback in controlling the center of foot pressure displacement in quiet standing in elderly subjects. Arch Phys Med Rehabil 2004;85:1962-1965.

Veale JF. Edinburgh Handedness Inventory - Short Form: a revised version based on confirmatory factor analysis. Laterality 2014;19:164-177.

Wickham J, Pizzari T, Stansfeld K, Burnside A, Watson L. Quantifying 'normal' shoulder muscle activity during abduction. J Electromyogr Kinesiol 2010;20:212-222.

Yoshizaki K, Hamada J, Tamai K, Sahara R, Fujiwara T, Fujimoto T. Analysis of the scapulohumeral rhythm and electromyography of the shoulder muscles during elevation and lowering: comparison of dominant and nondominant shoulders. J Shoulder Elbow Surg 2009;18:756-763.

Yun X, Bachmann ER. Design, Implementation, and experimental results of a quaternion-based kalman filter for human body motion tracking. IEEE Trans Robot 2006;22:1216-1227. 